\title{
DEDICATED TO MEMORY OF PROFESSOR ROBERT JANIN
}

\author{
J. M. RAKOTOSON
}

Mathematics subject classification (2010): 01A70.

\section{REFERENCES}

[1] R. JANIN Conditions nécessaires d'optimalité dans un problème d'optimisation en horizon infini, CRAS, série A-B 28913 (1979), A651-A653.

[2] J. GAUVIN Directional derivative of the value function in parametric optimization, Annals of Operations Research, December 1990, Volume 27, Issue 1, p 237-252. 